\title{
Methods for model generation and parameter extraction for MEMS
}

\author{
Peter Voigt, Gabriele Schrag and Gerhard Wachutka \\ Institute for Physics of Electrotechnology \\ Munich University of Technology \\ Arcisstr. 21, D-80290 München, Germany
}

\begin{abstract}
A strategy for compact modeling is presented, that takes into account the specific problems associated with the development and production of microelectromechanical devices and systems (MEMS). For a MEMS compact model, a set of basis functions is derived from a continuous field model description by proper approximations. This set should represent all relevant coupling effects that determine the device operation, and the most important parasitic effects. The remaining less important effects are taken into account by means of data fitting. The corresponding model parameter extraction process uses direct extraction techniques and well tuned local optimization steps for those parameters of the basis functions, which represent physical, technological or geometrical properties. Local and global optimization steps are used to determine the remaining fit parameters. This results in an effort-optimized modeling approach, which is suitable for statistical modeling and yield analysis, and can be automated in a CAD-toolset.
\end{abstract}

\section{Motivation}

There are several aspects in which microsystems technology differs vastly from integrated circuit (IC) technology. Integrated circuits are composed of a quite limited number of clementary device structures, fabricated by means of well established and quasi-standardized design rules and process technologies. In the field of microsystem technology, however, an ever growing variety of different device types has emerged, based on rather unconventional design methods and a large number of widely differing (and sometimes brandnew) fabrication technologies. Hence, today's challenge in the computer-aided IC design consists in mastering very complex system topologies built up by a huge number of simple basic elements, whereas in the computer-aided design of microelectromechanical systems we face the problem of describing systems with simple topology built up by a comparably small number of constituent devices which, however, exhibit a high functional complexity based on quite sophisticated and involved physical operating principles.

The complexity of microsystems originates in particular from the often complicated coupling between different energy and signal domains which, on the one hand, is the intierent and much desired property of any sensor or actuator element in a microsystem and, on the other hand, is a detrimental property when it occurs as parasitic cross coupling between the system components. Therefore the accurate analysis of all kinds of physical coupling 
effects has a major impact on the optimization of microsystems and is thus the most important issue that has to be tackled in the computer-aided design of microtransducers and microsystems. In this sense, the physical-based modeling of microsystems is widely recognized as necessity, even though it becomes increasingly complex, so that the effort as well as the time spent into model development, validation and parameter extraction have to be carefully adjusted to the actual needs, as it has been proposed by the concept of "tailored modeling" [1].

As the state of the art in modeling, simulation, and design optimization is far advanced in the world of microelectronics, it makes sense to revisit the well-tried methodologies for model generation and parameter extraction used in IC technology. In this context, the particular demands on the models must be identified which arise from their dedicated application to microsystems. This will provide us with decision criteria what modeling approach is most appropriate for a given problem and, consequently, should be implemented in a CAD toolbox for MEMS, which then will allow for an efficient and time-economizing computer-aided microsystem development.

\section{Model development}

The most rigorous approach to develop a device model is a basic physical analysis of its operation principles. Starting from a continuous field model (CFM) description, the degrees of freedom in the model have to be reduced by proper approximations. This results in a compact model that still reproduces all important physical effects of the device operation correctly, but allows, due to its relative simplicity, the simulation of the device behavior on the system level [1]. The resulting model equations contain parameters, which represent physical, geometrical or technological quantities. They can serve as basis functions and, with the help of additional fit parameters, are used to reproduce the device characteristics. However, for various reasons (complexity of the device geometry, complicated coupling effects) it may turn out impossible to consequently follow this way.

A possible alternative then is the numerical simulation of the device behavior by direct use of the original CFM model, employing FEM tools, for instance. This approach yields accurate information about the operation principles of the device, but the FEM model needs to be set up and interpreted carefully. Otherwise, important effects may easily be overlooked. The required effort ranges from moderate to prohibitive, depending on the device complexity and the availability of adequate software. Notably the coupling effects require special numerical methods.

The easiest way to generate compact models is pure curve fitting, based on measured data or on CFM simulation results. Such a model, if not supported by a physical description of the device operation, lacks all predictive capabilities and can hardly be used to inter- or extrapolate in the space of design parameters and operating conditions.

The choice for the optimum modeling approach depends on technological contraints, the system application of the device, and other factors. If a device is realized in just one version using one given technology, the model may be based on curve fitting procedures. But if a device would have many variants (geometry variations, e.g.), then a generic model is required that correctly reproduces the dependences from technology parameters, operating conditions, geometry etc.

In most practical cases, modeling is an overlap of physical considerations, CFM-based problem analysis, and data fitting. An instructive example is the model of an electrostatically actuated pump membrane (presented in [2]). It demonstrates a compromise between cost and benefits in the modeling process. The electrostatic force between membrane and 

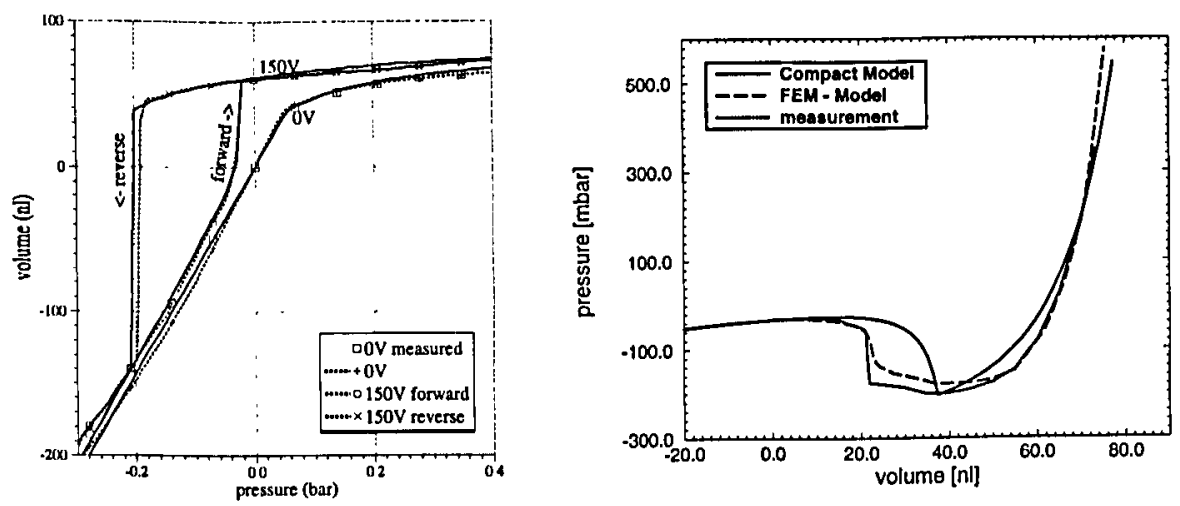

Figure 1: Static characteristics of an electrostatically deflected membrane. The left figure shows volume deflection vs. pressure. The right figure shows the resulting pressure, when volume is forced. A voltage of $150 \mathrm{~V}$ is applied to the membrane, causing the snap effect.

rigid counterelectrode is modelled physically with only little, well justified approximations. This treatment reproduces correctly the hysteresis phenomenon of the membrane snap-down due to an applied voltage. The shape of the membrane deflection, however, is simply fitted by an sinusoidal function. The effect of shape snapping, as calculated by FEM, is not included in the compact model and therefore cannot be reproduced (see Fig. 1). It is the responsibility of the modeling engineer to decide if this approximation is sufficient for the system applications in mind.

Other examples of such a mixed approach are given in [3], [4] and [5], among others. Here the device behavior is described by linear combinations of appropriately chosen basis functions. These functions are derived from an analytical approximation of the device and/or CFM and, therefore, represent to a certain extent the physical properties of the device. This way of model generation is amenable to automation in a dedicated software environment.

\section{Model verification and parameter extraction}

Model development cannot be considered independently of model validation and parameter extraction. Each modeling approach requires an appropriate extraction technique and, in return, the limitations of different extraction methods may influence the decision for a specific modeling strategy.

In case of a modeling strategy based on data fitting, the model verification is simply a test if the simulated data reproduces the input data from measurement or CFM simulation within the required accuracy. The appropriate extraction technique would be a global optimization algorithm. The advantage is a very fast setup of the extraction, but one pays with typically lengthy optimization runs. The extracted parameters can hardly be used for statistical process monitoring and a physical interpretation is quite often impossible.

If the developed model is based on the physical analysis of the device, a global optimization scheme could easily erode the physical content of the model parameters. Therefore, an extraction scheme utilizing direct extraction steps and carefully tuned local optimization needs to be employed. Only the remaining fit parameters can be extracted by means of global optimization. Model validation becomes a very challenging task since it must be 
verified, if the model reproduces all relevant physical effects together with their process and geometry dependence. In that case, both steps, model validation and extraction setup, are very time consuming, but the resulting model parameters can be used for physical interpretation, yield analysis, process monitoring, etc. The time required for an actual parameter extraction run is typically short, thus allowing the processing of a large amount of data as it is required for statistical analysis.

The parameters with a physical meaning are (more or less) independent from an actual device structure, thus allowing to extract them by measuring dedicated test structures. A parameter database may be established, that makes simulations possible in an early development stage, before real devices exist.

\section{Conclusions}

A synopsis of the special demands on MEMS device models including the strengths and weaknesses of alternative modeling and parameter extraction techniques led us to the conclusion that a hybrid modeling approach is best suitable in most situations. This approach uses basis functions to fit the device characteristics. These basis functions represent an approximation of the underlying physical effects of the device operation and can be derived analytically from CFM analysis. Their advantage compared to pure fit functions is the fact that they contain 'physical functionality'. The adequate parameter extraction process utilizes direct extraction and local optimization steps for all physically based parameters and global optimization for the fit parameters. Once a library of device classes and their corresponding basis functions have been developed, the modeling and parameter extraction process can be automated, thereby minimizing the required development effort. The suggested modeling approach is suitable for process monitoring and statistical analysis.

\section{References}

[1] G. Wachutka, "Tailored modeling: a way to the 'virtual microtransducer fab'?", Sensors and Actuators, vol. A46-47, pp. 603-612, 1995.

[2] P. Voigt, G. Schrag, and G. Wachutka, "Micropump macromodel for standard circuit simulators using HDL-A", in Proc. of the 10th European Conf. on Solid-State Transducers (EuroSensors X), R. Puers, Ed., Leuven, 1996, pp. 1361-1364, Timshel BVBA, Leuven.

[3] E. S. Hung, Yao-Joe Yang, and S. D. Senturia, "Low-order models for fast dynamical simulation of MEMS microstructures", in Dig. of Tech. Papers of Transducers'97, Chicago, June 16-19, 1997, pp. 1101-1104.

[4] S. Kurth and W. Dötzel, "Experimental adaption of model parameters for microelectromechanical systems (MEMS)", Sensors and Actuators, vol. A62, pp. 760-764, 1997.

[5] N.R. Swart, S.F. Bart, M.H. Zaman, M. Mariappan, J.R. Gilbert, and D. Murphy, "AutoMM: Automatic generation of dynamic macromodels for MEMS devices", in Proceedings of MEMS'98, Heidelberg, 1998, pp. 178-183. 